\title{
Prescribing adrenaline auto-injectors in Slovenian children
}

\author{
Tina Vesel ${ }^{1 *}$, Anja Koren Jeverica', Metka Accetto ${ }^{1}$, Natasha Toplak', Marijana Kuhar ${ }^{1}$, Vesna Glavnik', \\ Stefan Blazina', Gašper Markelj', Mirjana Zupančič ${ }^{1}$, Mira Šilar ${ }^{2}$, Peter Korošec ${ }^{2}$, Tadej Avcin ${ }^{1}$ \\ From Food Allergy and Anaphylaxis Meeting 2014 \\ Dublin, Ireland. 9-11 October 2014
}

\section{Introduction}

Little is known about the reasons for prescribing adrenalin auto-injectors in Slovenian children. Our objective was to evaluate prescription of adrenaline auto-injectors in children in our department in year 2013 and also to evaluate management of anaphylaxis of those children.

\section{Methods}

We retrospectively collected data on interventions of anaphylaxis and allergic-clinic follow-up of 258 children which had adrenaline auto-injectors prescribed in year 2013.

\section{Results}

Adrenaline auto-injector was prescribed in 258 children (66\% boys and $34 \%$ girls). In 120 of them adrenaline auto-injector was prescribed de novo. $2 \%$ of them were babies, $31 \% 1$ to 5 years old, $52 \% 6$ to 14 years old and $15 \% 15$ to 18 years old. In $66 \%$ adrenaline auto-injector was prescribed because of anaphylaxis, in $27 \%$ because of urticaria or/and angioedema and in $7 \%$ because of other reasons such as food allergy and bronchial asthma or/and recombinant based IgE testing results.

In 174 children (67\%) adrenaline auto-injector was prescribed because of food allergy, most frequently because of allergy to peanuts ( 81 children), egg ( 35 children), tree nuts ( 19 children) and cow milk (14 children). $77 \%$ of peanut allergic children had IgE antibodies to rArah $h 2$. Among food allergic children $44 \%$ had multiple food allergies, $40 \%$ asthma and $11 \%$ suffered more than one immediate reaction. In 60 children (23\%) adrenaline auto-injector was prescribed because of insect sting (49 had anaphylaxis and eleven urticaria or/and angioedema).
Allergy to wasp or hornet venom was more frequently confirmed (in 34 children) than allergy to honeybee venom ( 23 children). 3 children had negative testing results after anaphylaxis due to a sting. In four children parents refused starting specific immunotherapy with Hymenoptera venom after anaphylaxis. In 12 children (5\%) the cause of immediate reaction was unknown. Other confirmed reasons for prescribing adrenaline autoinjector were rare (such as cold, inhalant allergens, latex).

$30 \%$ of anaphylaxis was treated with adrenaline (autoinjector was used in eight children). $23 \%$ of children were not admitted to hospital after anaphylaxis. No child was admitted to intensive care unit.

\section{Conclusions}

Our data showed that allergy to peanuts was most frequent cause of prescribing adrenaline auto-injector to children during last year. There is need to increase education of management of anaphylaxis in children in Slovenia.

\section{Authors' details \\ 'University Children's Hospital, University Medical Center, Ljubljana, Slovenia.} ${ }^{2}$ University Clinic of Respiratory and Allergic Diseases, Golnik, Slovenia.

Published: 30 March 2015

doi:10.1186/2045-7022-5-S3-P109

Cite this article as: Vesel et al:: Prescribing adrenaline auto-injectors in Slovenian children. Clinical and Translational Allergy 2015 5(Suppl 3):P109.

'University Children's Hospital, University Medical Center, Ljubljana, Slovenia Full list of author information is available at the end of the article 